Acta vet. scand. 1990, 31, 493-495.

Brief Communication

\title{
Demonstration of Pasteurella multocida Type 6:B (B:2) in Formalin-Fixed Paraffin-Embedded Tissues of Buffaloes by the Peroxidase Anti-Peroxidase (PAP) Technique
}

Pasteurella multocida type $6: \mathrm{B}$ is the specific serotype of the genus Pasteurella responsible for the cause of haemorrhagic septicaemia (HS), a highly fatal, septicaemic disease in cattle and buffaloes in Asia. The disease is endemic in almost all South East Asian countries and is ranked as the major cause of mortality in buffaloes, a species of vital importance in the rural economy of the region. Several authors (Carter \& De Alwis 1989, Losos 1986, De Alwis 1984) have extensively reviewed the literature on HS. Reference has been made to a carrier status in HS and the dearth of information on the pathology of this infection.

Recent studies by De Alwis et al. (1990) have demonstrated the persistence of the organism in the tonsil of HS carriers for prolonged periods, in one instance extending upto 229 days. Studies on the pathology of HS in the buffaloes have been conducted and preliminary findings have been reported (Horadagoda et al. 1989). In order to pursue the findings of the afore mentioned studies on the carrier status and the pathological investigations, it was decided to examine the possibility of using an immunolabelling technique to determine the distribution of the organism in tissues.

In this report we describe the use of a rabbit polyclonal antibody for immunohistological detection of $P$. multocida type 6:B antigens in formalin-fixed, paraffin-embedded tissues from the buffalo.
Tonsils from experimentally induced HS carriers (Horadagoda et al. in prep.) and lungs, kidney, intestines and liver from animals that died following experimental infection were tested. The presence of $P$. multocida in these tissues was confirmed by isolating the organism according to the method described by De Alwis (1989). Tissue samples from noninfected buffalo calves and tonsils collected from cattle calves at the Uppsala slaughter house were used as negative controls. All tissues were fixed in $10 \%$ neutral buffered formalin and embedded in paraffin according to routine histological procedures. Sections were deparaffinised and rinsed in $0.5 \mathrm{~mol} / \mathrm{l}$ Tris- $\mathrm{HCl}$ buffer, $\mathrm{pH} 7.6$ containing $0.15 \mathrm{~mol} / \mathrm{l} \mathrm{NaCl}$ (TBS). Endogenous peroxidase was inactivated by incubating sections for $20 \mathrm{~min}$ in TBS containing $0.3 \%(\mathrm{v} / \mathrm{v})$ hydrogen peroxide. The sections were then rinsed thoroughly in TBS and incubated for a further $10 \mathrm{~min}$ with $2 \%$ BSA in TBS buffer. A hyperimmune antiserum to Pasteurella multocida type 6:B raised in rabbits, essentially according to the procedure described by Carter (1984) was used as the primary antibody. The sections were incubated for $45 \mathrm{~min}$ with a 1:5000 dilution of the Pasteurella antiserum followed by $30 \mathrm{~min}$ incubation with swine antirabbit IgG and rabbit PAP-complex (DAKOPATTS, Glostrup, Denmark) diluted $1: 20$ and $1: 100$, respectively. All dilutions were made in TBS containing $1 \%$ BSA; the 


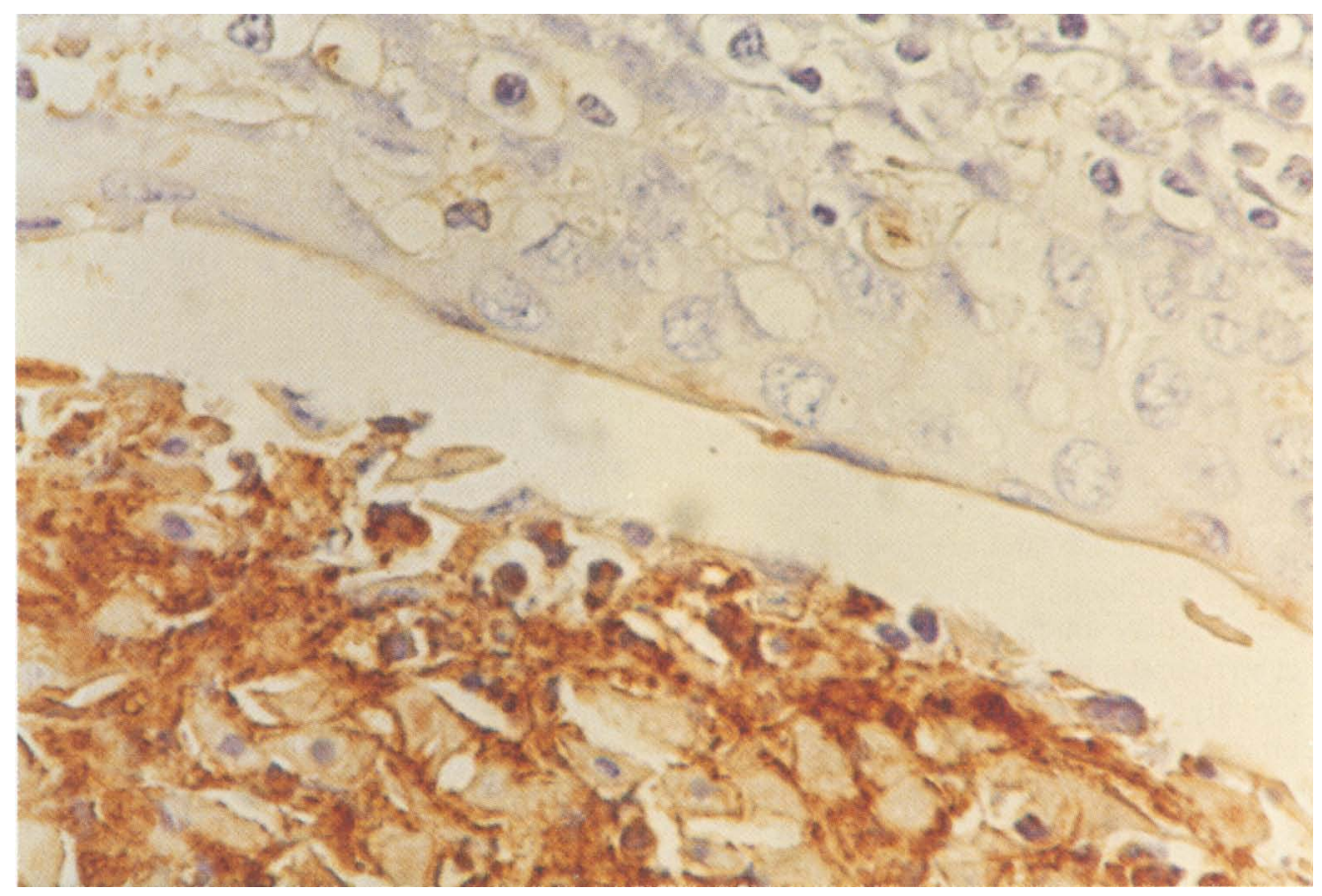

Figure 1. Immunostaining for $P$. multocida revealing bacterial antigen in the lumen of the tonsillar crypt. $\times 250$.

sections were thoroughly rinsed in TBS between each incubation. Peroxidase activity in tissues was visualised by incubation sections for $8 \mathrm{~min}$ in TBS containing $0.06 \%$ $(w / v)$ diaminobenzidine (DAB, Sigma, St. Louis, USA) and $0.034 \%(\mathrm{v} / \mathrm{v})$ hydrogen peroxide. Finally, the sections were rinsed in tap water, counterstained in Mayer's haematoxylin and mounted with Entellan (Merck, Darmstadt, F.R.G.).

Fig. 1 shows a typical positive staining reaction of the tonsil in a HS carrier. Antigens to $P$. multocida were confined to the lumen of the tonsillar crypt while the lining epithelium and lymphoid tissues were completely devoid of any immunoreaction.

In the lungs of animals that succumbed to the infection diffuse immunostaining was present in alveoli with concentration within alveolar macrophages. Bacterial antigen was also present in lymphatics of the interlobular septae and pleura. In the kidneys, intestines and the liver staining was present exclusively in the blood vascular system indicating the terminal septaemia associated with HS. Immunostaining was neither observed in tissues from non-infected buffaloes nor in cattle tonsils collected at the Uppsala abattoir.

The results were negative when the nonimmune rabbit serum was used as primary antibody on sections from infected animals. Immunoreactions were not present in infected tissues when the primary antibody was preabsorbed with the organism $\left(1.6 \times 10^{10}\right)$ prior to it been used in the staining proce- 
dure. Crossreactivity of the rabbit antiserum with other species of Pasteurellae was tested by incubating bovine and porcine lung from which P. haemolytica and P. multocida respectively, has been isolated. Murine tissues positive for $P$. pneumotropica were also tested. Staining was not observed in the bovine or murine sections but the porcine lung did show immunostaining. Crossreactivity of the primary antiserum may be expected since we used a polyclonal antibody raised against the entire organism.

This crossreaction may also underline the close similarities of the antigens present in the two types of Pasteurella. As far as the present study is concerned, the tissues tested were from experimentally infected animals with HS caused by $P$. multocida. Moreover, $P$. multocida species affecting pigs do not produce HS in buffaloes (Carter 1967).

The results of this study indicate that the PAP technique is a suitable immunochemical procedure for locating $P$. multocida in tissue sections. This method also offers the opportunity to simultaneously appreciate the histopathological changes associated with the infection.

\section{Acknowledgements}

The authors wish to thank the Swedish Agency for Research Cooperation with developing Countries (SAREC) for financial assistance. Prof. C. Rehbinder is gratefully acknowledged for critically evaluating the results. We also wish to express our gratitude to Ms. Eva Westergren, Ms. Cajsa Magnusson and Mr. Bengt Ekberg for technical assistance; Dr. E. Rivera for assistance in numerous ways during the investigations.
Neil Horadagoda and Katinka Belák

Faculty of Veterinary Medicine and Animal

Science, University of Peradeniya, Sri Lanka;

Department of Pathology,

National Veterinary Institute, Uppsala, Sweden.

\section{References}

Carter GR: Pasteurellosis: Pasteurella multocida and Pasteurella haemolytica. Adv. vet. Sci. 1967, 11, 321-368.

Carter GR: Serotyping of Pasteurella multocida. In: Methods in Microbiology. 1984, 16, 247258.

Carter GR, De Alwis MCL: Haemorrhagic septicaemia. In: Pasteurella and Pasteurellosis. Academic Press, London 1989, p. 131-160.

De Alwis MCL: Haemorrhagic septicaemia in cattle and buffaloes. Rev. Sci. Tech. Office Int. Epizooties 1984, p. 707-730.

De Alwis MCL: Haemorrhagic septicaemia. In: Manual of recommended diagnostic techniques and requirements for biological products for list A \& B diseases. 1989, OIE Manual Vol. I, 1-8.

De Alwis MCL, Wijewardena TG, Gomis AIU, Vipulasiri AA: Persistence of the carrier status in haemorrhagic septicaemia (Pasteurella multocida serotype 6:B infection) in buffaloes. Trop. Anim. Health Prod. 1990, in press.

Horadagoda NU, De Alwis MCL, Gomis AIU, Vipulasiri AA, Molligoda SC: Experimental Haemorrhagic Septicaemia; Clinical, Bacteriological and Pathological Studies. Symposium on Buffalo Research in Sri Lanka 1989, p. 46 $-48$.

Losos GJ: Haemorrhagic Septicaemia. In: Infectious Tropical Diseases on Domestic Animals. Longman Scientific \& Technical 1986, p. 719 -738 .

(Accepted June 13, 1990).

Reprints may be requested from: Katinka Belák, Department of Pathology, National Veterinary Institute, P. O. Box 7073, S-75007 Uppsala, Sweden. 